\title{
SUR UN CAPILLARIA DU VANNEAU
}

\author{
Par Alain G. CHABAUD
}

La détermination des Capillaria parasites du Vanneau est difficile, car certaines espèces ne sont connues que par des descriptions très anciennes; nous croyons donc qu'il est utile de décrire le matériel dont nous disposons, bien qu'il soit peu abondant.

Matériel. - Une femelle mature a été récoltée sous la membrane du gésier, un mâle et deux larves dans l'eau de rinçage de l'œsophage et de l'intestin, au cours de la dissection d'un Vanellus vanellus (L.), en mars 1952. L'oiseau acheté à Paris, a, au dire du marchand, été tué dans le département du Loiret.

Description. - Femelle longue de $31,7 \mathrm{~mm}$. ; portion œsophagienne longue de $4,1 \mathrm{~mm}$., large de $8 \mu$ en avant et de $65 \mu$ à la base ; portion postérieure longue de $27,6 \mathrm{~mm}$., large de $100 \mu$. Il existe une très grosse bande bacillaire, large de $30 \mu$ dans la région vulvaire, de $48 \mu$ dans la région caudale, qui se manifeste à la surface de la cuticule par un aspect régulièrement criblé. Cette bande occupe, dans la région vulvaire, la zone qui sépare la ligne latérale droite et la ligne dorsale ; il est difficile de préciser sa position sur le reste du corps, car l'anus est strictement terminal et ne peut indiquer l'orientation du corps. En dehors de cette bande bacillaire bien marquée, il existe trois zones où l'hypoderme est noirâtre ; la bande gauche est large de $10 \mu$ dans la région vulvaire et de $22 \mu$ dans la région caudale; les deux bandes latérales ne sont distinctes que dans la région caudale et restent très étroites $(3 \mu)$.

Cuticule épaisse à stries transversales espacées de $3 \mu$. Préœesophage long de $600 \mu$. La vulve, située $200 \mu$ en arrière de la fin de l'œsophage, est dépourvue de lèvres saillantes et se trouve marquée simplement par une fente longitudinale qui s'ouvre sur une surface plane, ovalaire, longue de $60 \mu$ et large de $28 \mu$. Le vagin est long de $900 \mu$. L'utérus contient des œufs alignés sur une file simple ou

Ans. de Parasitologie, T. XXVII, N $4 .-1952$. 
double ; l'oviducte fait une boucle à concavité antérieure à $12 \mathrm{~mm}$. de la pointe caudale ; l'extrémité postérieure de l'oviducte n'a pas été repérée avec certitude. La pointe caudale est arrondie et l'anus est exactement terminal. Les œufs ont un aspect très comparable

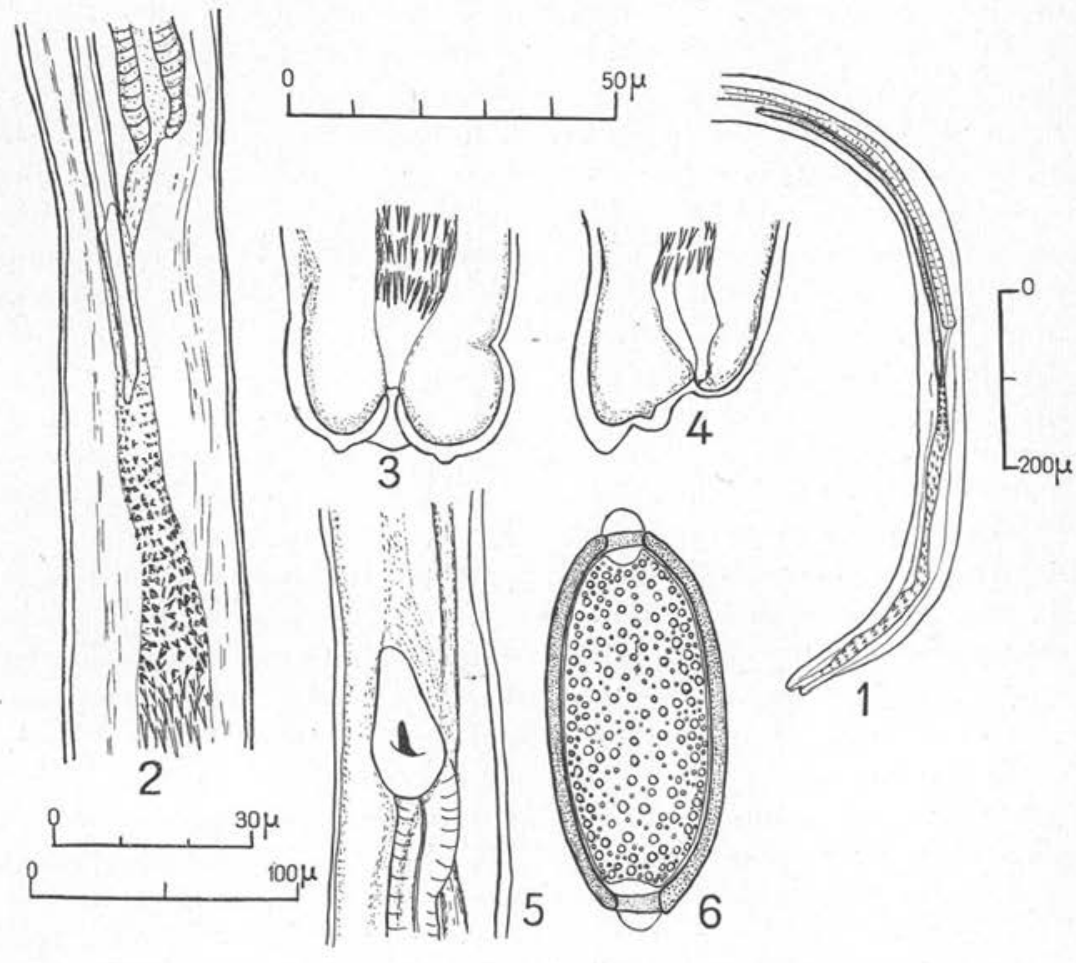

Capillaria vanelli (Rud.)

Fig. 1. - Extrémité postérieure du mâle ; vue latérale

Fig. 2. - Détail du fond de la gaine et de la portion distale du spicule

Fıg. 5. -- Pointe caudale du mâle; vue ventrale

FIG. 4. - Pointe caudale du mâle; vue latérale

Fig. 5. - Région vulvaire; vue ventrale

Fig. 6. - Euf.

1 échelle, $0-200 \mu$; 2 échelle, $0-30 \mu$;

3-4-6 échelle, 0-50 н; 5 échelle, $0-100 \mu$

à ceux du Capillaria contorta (Creplin 1839) et sont également munis à chaque pôle d'un bouchon très transparent. Ils mesurent 56 à $60 \mu$ de long (sans compter les bouchons), ou 65 à $70 \mu$ (en comptant les bouchons), et sont larges de 25 à $28 \mu$. 
Mâle long de 9,6 mm., portion œsophagienne longue de 3,1 mm., large de $5 \mu$ en avant et de $40 \mu$ à la base ; portion postérieure longue de 6,5 mm., large de $45 \mu$. La bande bacillaire principale est latéro-droite ; elle est large de $27 \mu$ dans la région œsophagienne et de $20 \mu$ dans la région caudale. La bande bacillaire diamétralement opposée à la précédente est beaucoup moins marquée ; elle est large de $10 \mu$ dans la région œsophagienne et disparaît dans la région caudale. Cuticule épaisse, à stries transversales espacées de $2 \mu$. Le testicule se replie un peu en arrière de la fin de l'œsophage ; la limite entre canal déférent et canal éjaculateur est marquée par un gros sphincter à $1,35 \mathrm{~mm}$. de la pointe caudale ; la portion musculeuse du canal éjaculateur se termine postérieurement à $580 \mu$ de la pointe caudale. La queue est formée par deux lobes latéraux du corps terminés chacun par une papille et reliés entre eux dorsalement par une membrane transparente. La gaine du spicule est entièrement invaginée ; longue dans son ensemble de $515 \mu$ et large de $18 \mu$, elle comprend trois parties bien distinctes ; une portion distale longue de $380 \mu$ est couverte d'épines sétacées fines et allongées, à pointe postérieure ; une portion proximale haute de $85 \mu$, qui correspond probablement à l'ampoule lorsque l'appareil est évaginé, se distingue par des épines fortement chitinoïdes, courtes et épaisses. Le sommet de cette zone spinulée est coiffé par une membrane épaisse sur laquelle se voient encore quelques très fines épines. Cette membrane a la forme d'un cône haut de $50 \mu$ et paraît former le fond de la gaine du spicule. Le spicule lui-même, bien que faiblement chitinoïde, se voit facilement sur l'animal vivant. Il est long de $390 \mu$ et large de $6 \mu$; sa pointe distale est mousse et se trouve sur notre exemplaire dans la portion antérieure de la zone spinulée.

Discussion. - Madsen (1945) a donné la liste des Capillaria signalés chez les Charadrii en général et chez Vanellus vanellus en particulier, et il nous faut comparer nos spécimens à ces différentes espèces.

C. charadrii (Rud. 1819) est un nomen nudum, ainsi que l'a souligné Travassos en 1915, et ce nom ne pourra être employé à nouveau que si l'on découvre chez les mêmes hòtes une espèce qui soit nettement distincte des formes suivantes.

C. totani (v. Linstow 1875) (1) [= Capillaria breve (v. Linstow 1877)]. Lopez-Neyra (1947) a rassemblé en une seule espèce un

(1) Madsen (1951) a montré que la division des Capillaria en différents genres, proposée par Lopez-Neyra (1947), est susceptible d'amener des erreurs importantes, et nous garderons ici l'ancienne non:enclature, qui ne comprend quiun seul genre. 
mâle et une femelle décrits successivement par v. Linstow. Le mâle se distingue de celui de rotre matériel par un spicule relativement beaucoup plus long, la femelle par sa petite taille et par la faible longueur des œufs. L'espèce n'est pas signalée chez le Vanneau.

C. obtusinscula (Rud. 1819). L'espèce se distingue de toutes celles qui nous intéressent ici par une gaine du spicule dépourvue d'épines. Ŗead (1949) a souligné que ce caractère pouvait prêter à confusion, mais dans le cas présent, on possède une figure de Mehlis (1831) qui montre la gaine évaginée èt il semble donc qu'on puisse s'appuyer sur cet élément pour distinguer l'espèce.

C. vanelli (Rud. 1819) étant un nomen nudum, Lopez-Neyra (1947) l'a employé pour désigner le $C$. protracta (Dujardin 1845), connu seulement par une femelle récoltée chez le Vanneau ; C. protracta se trouve ainsi placé en synonymie. Cette espèce est décrite comme provenant de l'intestin du Vanneau, mais la récolte n'a pas été faite par Dujardin et, étant donné la concordance parfaite qui existe entre la description originale et notre propre spécimen, nous croyons pouvcir négliger cet élément, et affirmer l'identité de notre exemplaire avec $C$. vanelli (Rud. 1819).

C. contorta (Creplin 1839). Lopez-Neyra (1947) a cherché à démembrer l'espèce classique en attribuant les récoltes faites chez des oiseaux autres que les Passériformes à des espèces distinctes. Ainsi, il n'admet pas l'existence de $C$. contorta chez les Charadriiformes et les nombreuses références de cette espèce chez les oiseaux de ce groupe sont attribuées tantôt à $C$. vanelli (Rud. 1819), tantôt à C. triloba (v. Linst. 1875). L'hypothèse de Lopez-Neyra est très séduisante, et il est possible que des études de biométrie statistique montrent le bien-fondé de cette théorie. Actuellement, il nous est malheureusement impossible de suivre cette classification, pour les raisons suivantes : $a$ ) en dehors d'anciennes références sujettes à caution, la présence de $C$. contorta chez des Charadrififormes a été signalée récemment par des auteurs dignes de foi ; par exemple, Madsen (1945), qui connaît particulièrement bien C. contorta, donne comme hôte nouveau pour cette espèce Erolia alpina (L.) ; $b$ ) nous verrons plus bas que $C$. vanelli (Yamaguti 1935) ne peut être attribué à $C$. vanelli (Rud. 1819), comme l'a fait Lopez-Neyra et doit être attribué à $C$. contorta. Nous devons donc admettre l'existence de C. contorta chez les Charadriiformes.

En cherchant à différencier nos spécimens à l'aide des principaux caractères choisis par Madsen, nous obtenons le tableau suivant : 


\begin{tabular}{|c|c|c|}
\hline . & C. vanelli (Rud, 1819) & C. contorta (Crep. 1£39) \\
\hline 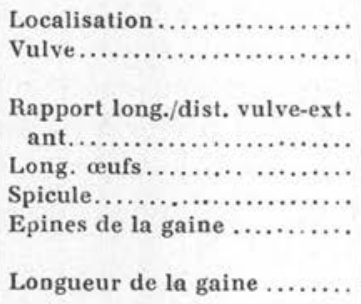 & $\begin{array}{c}\text { Gésier } \\
\text { Fente sur une grosse pla- } \\
\text { que ovalaire } \\
7,4-8 \\
58 \mu \\
\text { Bien visible; large de } 6 \mu \\
2 \text { groupes différents } \\
465 \mu\end{array}$ & $\begin{array}{l}\text { Esophage ou proventricule } \\
\text { Petite et circulaire } \\
2,5-6,6 \\
\text { moyenne } 54 \mu(1) \\
\text { Peu chitinisé ; large de } 3 \mu \\
\text { Peu différentes les unes des } \\
\text { autres } \\
760-6500 \mu\end{array}$ \\
\hline
\end{tabular}

La femelle possède donc de bons caractères susceptibles de la différencier facilement. La diagnose du mâle apparaît difficile, car chacun des éléments peut prêter à critique : $a$ ) le spicule est plus facilement visible sur les petits spécimens, ainsi que l'a constaté Cram (1936) ; b) Cram et Madsen ont signalé l'existence de quelques exemplaires où les épines de la portion antérieure de la gaine (invaginée) étaient plus grosses et plus nettes que les épines postérieures ; cette différenciation en deux zones n'est donc pas parfaitement caractéristique puisqu'elle se trouve déjà ébauchée chez certains spécimens de $C$. contorta ; $c$ ) la gaine du spicule de contorta, longue de $760 \mu$ au minimum, paraît à première vue un bon caractère puisque Madsen a étudié des exemplaires dont la longueur totale n'excédait pas 6,1 mm., mais Travassos (1915) et Lopez-Neyra (1947) parlent de gaine mesurant $300 \mu$.

Notre spécimen mâle n'ayant pas été récolté dans le gésier à côté de la femelle, il nous est donc impossible d'éliminer rigoureusement l'éventualité d'un pluriparasitisme chez notre oiseau : le mâle appartiendrait à contorta et la femelle à vanclli (Rud.). Cette coïncidence paraît cependant peu probabie et nous croyons simplement que les mâles de contorta et de vanelli (Rud.) sont très proches les uns des autres.

C. triloba (Linstow 1875), récolté dans le gésier du Vanneau, a été retrouvé par Skrjabin (1916), mais n'a pas été redécrit à ce moment. Les bandes bacillaires sont décrites par Linstow comme étant plus larges que dans nos spécimens, mais ce caractère est parfois difficile à apprécier et peut prêter à erreur. La description

(1) D. Orosz (Thèse, Budapest, 1931), cité par Freitas et Alineida (1935 b), downe des dimensions un peu plus grandes $(57$ à $65 \mu$ ), mais cet auteur a peutctre tenu compte des bouchons polaires. 
du mále correspond bien à notre matériel. La femelle est un peu plus courte et l'œsophage est relativement plus long, mais on connait chez les différentes espèces de Capillaria des variations intraspécifiques beaucoup plus larges. La dimension des œufs, enfin, peut également correspondre à notre matériel, si l'on tient compte dans la mensuration des deux bouchons muqueux polaires.

Il ne semble donc pas y avoir de différences morphologiques susceptibles d'avoir une valeur spécifique et nous pensons que C. triloba est synonyme de C. vantlli (Rud.).

C. vanelli (Yamaguti 1935), nec C. vanelli (Rud. 1819). Yamaguti a décrit sous ce nom une femelle récoltée chez Vanellus vanellus, qui est considérée comme espèce nouvelle. Freitas et Almeida (1935) et Madsen (1945) l'ont mise en synonymie de C. contorta ; LopezNeyra, au contraire, la considère synonyme de $C$. protracta, c'est-àdire de $C$. vanelli (Rud.). La description de Yamaguti indique des œufs longs de 50-53 $\mu$, une vulve petite et circulaire, un rapport longueur totale sur distance vulve-extrémité antérieure égal à 4,0. Il suffit de comparer ces données à celles qui figurent dans le tableau donné plus haut pour voir que l'espèce est synonyme de contorta et non de vanelli (Rud.).

Conclusion. - Notre matériel est trop peu abondant pour que nous puissions apporter des conclusions parfaitement rigoureuses. La femelle appartient certainement à l'espèce décrite par Dujardin, mais le mâle pourrait n'être qu'une forme atypique de $C$. contorta, bien que ce pluriparasitisme nous semble peu probable. Il serait donc utile de faire de nouvelles récoltes pour pouvoir affirmer que le mâle et la femelle, ci-dessus décrits, appartiennent bien à la même espèce.

Cette réserve étant faite, nous croyons que parmi toutes les espèces citées chez le Vanneau, il y a lieu de retenir seulement trois noms :

1. C. obtusiuscula (Rud. 1819), anciennement signalé dans le proventricule du Vanneau, mais dont les redescriptions (Mehlis 1831, Jägerskjöld 1901) portent seulement sur un matériel récolté chez Grus grus (L.).

2. C. vanelli (Rud. 1819) (= Trichosomum protractum, Dujardin $1845 ;=$ Trichosoma trilobum, v. Linstow 1875).

3. C. contorta (Creplin 1839) (= C. vanelli, Yamaguti 1935). 


\section{RÉsumé}

Description d'un Capillaria parasite de Vanellus vanellus, qui peut être assimilé au Trichosomum protractum de Dujardin. Revue des espèces citées chez le Vanneau. Mise en synonymie de $C$. triloba. Trois espèces seulement paraissent pouvoir être retenues : $C$. obtusiuscula (Rud. 1819), C. vanelli (Rud. 1819) et C. contorta (Creplin 1839).

\section{BIBLIOGRAPHIE}

Cram (E. B.). - Species of Cupillaria parasitic in the upper digestive tract of Birds. Tech. Bull. $n^{\circ}$ 516, U.S. Dept. Agr., 1936, 27 pp., 12 fig.

DUJARDIN (F.). - Histoire naturelle des helminthes ou vers intestinaux, xvI + 652 pp. + 12 pl., Paris, 1845.

Freitas (J. F. Teixeira) et A mmeida (J. L. DE). - Sobre os Nematoda Capillariinze parasitas de esophago e papo de aves. Mem. Inst. Osw. Cruz, $\mathrm{XXX}, 1935 a, 123-156+6 \mathrm{pl}$.

- $O$ genero «Capillaria \# Zeder 1800 (« Nematoda-Trichuroidea 》) e as Capillarioses nas aves domesticas. Rev. Depart. Nac. Prod. Ani., 1935 , $311-363+12 \mathrm{pl}$.

JîGerskïold (L. A.). - Weitere Beiträge zur Kenntnis der Nematoden, Svensk. Vet. Ak. Handl., XXXV, 1901, 1-80, + 6 pl., 8 fig.

Linstow (O. Von). - Beobachtungen an neuen und bekannten Helminthen. Arch. Naturg., XLI, 1875, 183-207, pl. 2-4.

Lopez-Neyra (C. R.). - Los Capillarinae. Mem. Real. Acad. Cienc. Madrid, XII, 1947,240 pp. $+17 \mathrm{pl}$.

Madsen (H.). - The species of Capillaria (Nematodes, Trichinclloidea) parasitic in the digestive tract of Danish gallinaceous and anatine game birds, with a revised list of species of Capillaria in birds. Danish Rev. of Game Biol.. I, 1945, 1-112, 22 fig.

- Notes on the species of Capillaria Zeder, 1800 known from gallinaceous birds. J. Parasit., 1951, 257-265.

MErrls (E.). - Novae observationes de Entozois. 1sis (Leipzig), I, 1831, 68$99+$ pl. 2, 18 fig.

REad (C. P.). - Studies on North american helminths of the genus Capillaria Zeder, 1800 (Nematoda), III. Capillarids from the lower digestive tract of North american birds. J. Parasit., XXXV, 1949, 240-247 +2 pl.

SkrJabin (K. J.). - Nématodes des oiseaux du Turkestan russe. Ann. Mus, Zool. Acad. Imper. Sci. Petrograd (1915), XX, 1916, 457-557, 1 pl., 45 fig.

Travassos (L.). - Contribuiçoes para o conhecimento da fauna helmintolcjica brasileira. V. Sobre as especies brasileiras do genero Capillaria Zeder, 1800. Mem. Inst. Osw. Cruz., VII, 1915, 146-172, pl. 23-26.

Yamaguti (S.). - Studies on the helminth fauna of Japan. Part. 12. Avian Nematodes, I. Jap. J. Zool., VI, 1935, 403-431, 41 fig.

Laboratoire de Parasitologie de la Faculté de Médecine de Paris (Directeur : Pr H. Galliard) 